\title{
An Audit of Prescribing Practices in CGHS Dispensaries of Kolkata, India
}

\author{
Amitabha Chattopadhyay 1, Tanushree Mondal 2, Tushar Kanti Saha 3, \\ Indira Dey 4,Badal Kumar Sahu 5, Jashodip Bhattacharya 6 \\ 1, (Assistant Professor, Department of Community Medicine, R.G.Kar Medical College ,West Bengal, India) \\ 2(Assistant Professor, ADME1, Govt of West Bengal, India) \\ 3, (Assistant Professor, Department of Community Medicine, NRS Medical College, West Bengal, India) \\ 4,(Associate Professor, Department of Community Medicine, NRS Medical College, West Bengal, India) \\ 5,6(Demonstrator, Department of Community Medicine, NRS Medical College, West Bengal, India)
}

\begin{abstract}
Background: Improved method of examining drug prescribing in a health service are a necessary prerequisite for planning measures intended to bring about financial economics in drug use with the least possible impairment of the quality of medical care. This study was therefore carried out to evaluate the prescribing pattern of General Duty Medical Officers (GDMO) of CGHS. Methodology: This health service research study, cross sectional in design was conducted over one year period in CGHS Dispensaries at Kolkata. A multistage sampling method was followed. Total number of respondents was 412. WHO/INRUD Core indicators were used in prescription analysis. Results: Average number of drugs per prescription in the study was 3.05+/-2.54(1-7). 33.0\% of all the prescriptions had antibiotics. Overall average consultation time was $2.3+/-1.53$ minutes and average dispensing time was $4.3+/-1.6$ minutes. E.D.L/formulary was available in all the selected dispensaries. Average cost per prescription was Rs.103.8+/-233. Conclusion: This study on prescription audit in CGHS Dispensaries at Kolkata revealed that prescribing habits of the G.D.M.O's as well as the dispensing practices may be bettered. There is a need to strengthen an independent mechanism for continuing professional development of practitioners to ensure that patients are always given evidence-based, cost-effective treatments.
\end{abstract}

Keywords - Prescription, audit, CGHS Dispensaries

Submitted date 19 June 2013

Accepted Date: 24 June 2013

\section{Introduction}

There is no global standard for medical prescriptions. Every country has its own standard regulations for the minimum information required for a prescription. Every laws and regulations regarding prescriptions define which drug requires a prescription and who is authorized to prescribe it.

The objective of prescribing is to encourage rational use of drugs i.e use in accordance with scientific knowledge to satisfy the needs. Prescribing is the most important area to be emphasized for rational drug use.

Rational prescriptions ensures maximum benefits with no/minimum ill-effects, cost optimization and sometimes good patient compliance also. Improved method of examining drug prescribing in a health service are a necessary prerequisite for planning measures intended to bring about financial economics in drug use with the least possible impairment of the quality of medical care. ${ }^{1-2}$

To assess the scope for improvement in rational drug use in outpatient practice, the World Health Organization (WHO) has formulated a set of "core drug use indicators". ${ }^{3}$ The core prescribing indicators measure the performance of prescribers, the patient care indicators measure what patients experience at health facilities, and the facility indicators measure whether the health personnel can function effectively.

This study was therefore carried out to evaluate the prescribing pattern of General Duty Medical Officers (GDMO) of Central Government Health Scheme (CGHS) Dispensaries with the aim of suggesting modifications in prescribing practices of medical practitioners so as to make medical care rational and cost effective services.

\section{Materials and Methods}

This health service research study, cross sectional in design was conducted over one year period (May 2008- April 2009) in CGHS Dispensaries at Kolkata. After necessary clearance from the ethics committee, a multistage sampling method was followed. Dispensaries were selected by simple random sampling method using random number table. Out of 18 CGHS Dispensaries at Kolkata, 5 CGHS Dispensaries (about 25\%) were 
selected .Patient selection was then done by systematic random sampling method. Every 5 th new patient on the day of data collection was selected. If the 5th patient refused to participate, the next patient was taken. Total number of patients approached was 431, out of which 19(4.4\%) refused to participate. Final total number of respondents was 412. Informed consent of the participants were taken; in case of respondents below $18 \mathrm{yrs}$ of age, parental consent was taken wherever available. Prescriptions of the selected respondents were copied in predesigned schedules during exit. Patient's knowledge of when and in what quantity each drug that was actually dispensed should be taken was evaluated by the dispensing survey schedule. Failure to know either of these two points resulted in patient's knowledge being judged as incorrect .Consultation time was taken as the time between entering and leaving the consultation room. Dispensing time was taken as the time between arriving at the dispensary counter and leaving. Waiting time was not included.

The features of WHO/INRUD Core indicators which were used in prescription analysis were -highly standardized, did not need national adaptation, did not measure all important aspects of drug utilization, was a simple tool for quick and reliable assessment of few critical aspects of pharmaceutical use in primary healthcare. WHO/INRUD CORE DRUG USE INDICATORS (recommended level in brackets) ${ }^{3}$

A) Prescribing indicators

- $\quad$ Average number of drugs per encounter $(<2)$

- $\quad$ Percentage of drugs prescribed by generic name (close to $100 \%$ )

- $\quad$ Percentage of encounters with an antibiotic prescribed $(<30 \%)$

- $\quad$ Percentage of encounters with an injection prescribed $(<10 \%)$

- Percentage of drugs prescribed from EDL or formulary (close to 100\%)

B) Patient care indicators

- Average consultation time

- $\quad$ Average dispensing time

- $\quad$ Percentage of drugs actually dispensed (100\%)

- $\quad$ Percentage of drugs adequately labelled (100\%)

- Patients knowledge of correct dosage (100\%)

C) Facility indicators

- Availability of copy of EDL or formulary (100\%)

- $\quad$ Availability of key drugs (100\%)

Complementary Indicators:

These are no less important, often more different to measure and cannot be collected reliably in some settings. These are less standardized and depend on local variables.

Data was entered in MICROSOFT EXCEL 8.0 software and was analyzed with the help of EPI INFO3.4.3 to obtain the results.

\section{Results}

Table 1 shows that average number of drugs per prescription in this study was 3.05+/-2.54(1-7). In total, 33.0\% of all the prescriptions had antibiotics, while $2.43 \%$ of the prescriptions had injectables in them. The total number of drugs prescribed in all the prescriptions selected was 1258, out of which 911(72.4\%) were prescribed from EDL or formulary, and only 131 (10.4\%) were prescribed by generic name.(Fig-1)

Table 2 shows that the overall average consultation time was 2.3+/-1.53 minutes and average dispensing time was $4.3+/-1.6$ minutes. $74.3 \%$ of the total number of respondents had proper knowledge about the correct dosage of the drugs prescribed; the number of drugs actually dispensed was 1019(81\%),out of which 1013(99.4\%)where adequately labeled.(Fig 2)

Table 3 shows that E.D.L/ formulary was available in all the selected dispensaries. \% of key drugs available was between 80 to $90 \%$ in the selected dispensaries.

Among the additional indicators, the average number of antibiotics per prescription in the study was $0.72+/-$ 2.27. (Table 4) In total only $8.7 \%$ of the prescriptions had proper history while only $8.3 \%$ of the prescriptions had diagnosis written in them. Overall \% of referrals was $14.3 \%$ while average cost per prescription was Rs.103.8+/-233.4.In total, $23.7 \%$ of the drugs were antibiotics among total number of drugs prescribed. The commonest antibiotic that was prescribed was cotrimoxazole $(16.1 \%$ of the total number of antibiotic prescribed). In total, only $10.2 \%$ of the prescriptions had dietary advices, while antiulcer drugs were present in about $30.1 \%$ of the prescriptions.

\section{Discussion}

The principal aim of drug utilization research is to facilitate rational use of drugs in populations. For the individual patient, rational use of a drug implies the prescription of a well-documented drug in an optimal dose on the right indication, with the correct information and at an affordable price. Without knowledge on how 
drugs are being prescribed and used, it is difficult to initiate a discussion on rational drug use and to suggest measures to change prescribing habits for the better.

A study was done on patterns of prescription and drug dispensing in a Medical College at Sion, Mumbai where the average number of drugs per encounter was 2.9 and $73.4 \%$ drugs were prescribed by generic name ${ }^{4}$. In a drug utilization review carried out in the medical outpatient department of the Manipal teaching hospital in Pokhara, western Nepal, the mean +/- SD drugs per prescription was $2.15+/-1.71 ; 67.4 \%$ of the drugs were prescribed by brand name and only $39.56 \%$ of the drugs prescribed were from the WHO essential drug list and the frequency of prescribing antibiotics was $23.96 \% .5$ In our study the average number of drugs per encounter was high $3.05+/-2.54$, percentage of drugs prescribed by generic name and percentage of drugs prescribed from the EDL was less than $100 \%(10.4 \% \& 72.4 \%$ respectively) and percentage of encounters with an antibiotic prescribed was more than $30 \%$.

A cross-sectional institutional observational study done at Medical College Hospital, Kolkata found an average consultation time of 127.99 +/- $21.9 \mathrm{sec}$. and average dispensing time of $49.13+/-8.45 \mathrm{sec} .51 .54$ percent of drugs were actually dispensed, 21.43 percent of drugs were adequately labelled, and only 44.67 percent of the patients knew the correct dosage of drugs given to them. ${ }^{6}$ The study done in Sion, Mumbai ${ }^{4}$ reported that parents of pediatrics patients $(80.8 \%)$ knew the correct dosages, but only $18.5 \%$ of drugs were adequately labeled. In the present study, the average consultation time was $2.3+/-1.53$ minutes and average dispensing time was $4.3+/-1.6$ mins. $74.3 \%$ of the total number of respondents had proper knowledge about the correct dosage of the drugs prescribed; the number of drugs actually dispensed was 1019(81\%),out of which 1013(99.4\%)where adequately labeled.

The optimal values for percentage of drugs actually dispensed, adequately labeled and the patients correct knowledge about dosage should be $100 \%$ for all as recommended by W.H.O. Results of this study was better than other studies, but was still not upto the recommended 100\% label. E.D.L/ formulary was available in all the selected dispensaries. Percentage of key drugs available was between 80 to $90 \%$ in the dispensaries. None of the dispensaries was having value up to the W.H.O recommended level of $100 \%$.

Among the additional indicators, $23.7 \%$ of the drugs were antibiotics among total number of drugs prescribed and the average number of antibiotics per prescription in this study was $0.72+/-2.27$ while in a study of drug prescribing pattern of interns at a government healthcare centre, ${ }^{7} 33.9 \%$ of the drugs prescribed were antibiotics and $13 \%$ of the drugs were vitamins. In a study undertaken by Salman MT e.tal, ${ }^{8}$ average number of antibiotics per prescription was 2.2 , while the average cost estimation per prescription was Rs.246. The average cost estimation per prescription in this study was Rs.103.8+/-233.4. The value of these indicators were lower than that in other studies most probably due to the fact that only G.D.M.O's prescriptions were selected for this study and inclusion of specialists prescriptions may have resulted in higher values. Only $8.3 \%$ of the prescriptions had diagnosis written in them in our study while the same in a study at Government Combined Hospital, Srinagar (Garhwal) ${ }^{9}$ was 22.2. Overall percentage of anti hypertensives drugs(27.4\%), antiulcer drugs $(30.1 \%)$, were higher in this study in comparison to other similar studies ${ }^{5}$. In general, the percentage of prescriptions in this study with proper history and diagnosis written was scanty; similarly fewer number of prescriptions had dietary advice in them.

\section{Conclusion}

This study on prescription audit in CGHS Dispensaries at Kolkata revealed that prescribing habits of the G.D.M.O's as well as the dispensing practices were inappropriate in some aspects. The prescribing indicators showed that the average number of drugs per encounter was high, percentage of drugs prescribed by generic name and percentage of drugs prescribed from the EDL was less than recommended value. Optimal values for patient care indicators like percentage of drugs actually dispense, adequately labeled and the patients correct knowledge about dosage was still less than the $100 \%$ value as recommended by W.H.O. The percentage of prescriptions in this study with proper history and diagnosis written was scanty thus there is a need to standardize the format of prescriptions in India so that all essential information is included. There is a need to strengthen an independent mechanism for continuing professional development of practitioners to ensure that patients are always given evidence-based, cost-effective treatments. Irrational prescribing is a habit that is difficult to cure. However, prevention is possible. There is some evidence that interventions such as short problem-based training course in pharmacotherapy and rational use focused workshops can improve prescription behavior and skills. There is an urgent need to implement training initiatives, with support from public sources to ensure that there is no conflict of interest, to improve prescription behavior of practitioners in India and ensure that patients receive evidence-based, cost-effective treatments for their health problems. Interventions are also required to improve prescribing by generic name so as to rationalize drug use in general population. 


\section{Acknowledgement}

Sincere gratitude to Late Prof.Ranadeb Biswas \& Prof. A.Dasgupta, Faculty, Dept of PSM,AIIH\&PH, Kolkata for their valuable suggestions in relation to this study.

\section{References}

[1] Managing Drug Supply-The selection, procurement, distribution and use of pharmaceuticals. West Hartford, M.S.H and W.H.O collaboration, Action programme on Essential drugs.1997.

[2] Halder D. An Assessment of Inventory Control and Drug Utilization Pattern in Central Government Health Scheme Dispensaries at Kolkata. Thesis; All India Institute of Hygiene and Public Health,2004.

[3] Hogerzeil Hans V. Promoting the rational use of medicines WHO Essential Drugs and Medicines Policy: October 2002.

[4] Karande S, Sankhe P, Kulkarni M. Patterns of prescription and drug dispensing. Indian J Pediatr 2005;72:117-121

[5] Ravi Shankar P, Partha P, Nagesh S. Prescribing patterns in medical outpatients. Int J Clin Pract 2002; 56: 549-551

[6] Biswas R; Chatterjee P; Mundle M; Prescribing habits of physicians in Medical College, Calcutta; Indian Journal of Community Medicine. 2000 Oct-Dec; 25(4): 161-5

[7] Rehan HS, Lal P. Drug prescribing pattern of interns at a government healthcare centre in northern India. Trop Doct 2002; 32 : 4-7.

[8] Salman MTe.tal. Drug Prescribing Pattern in Surgical Wards of a Teaching Hospital in North India. Indian Journal for the Practising Doctor :Vol. 5, No. 2 (2008-05 - 2008-06)

[9] Rishi RK, Sangeeta S, Surendra K, Tailang M. Prescription audit: Experience in Garhwal (Uttaranchal), India. Trop Doct 2003;33:76-9.

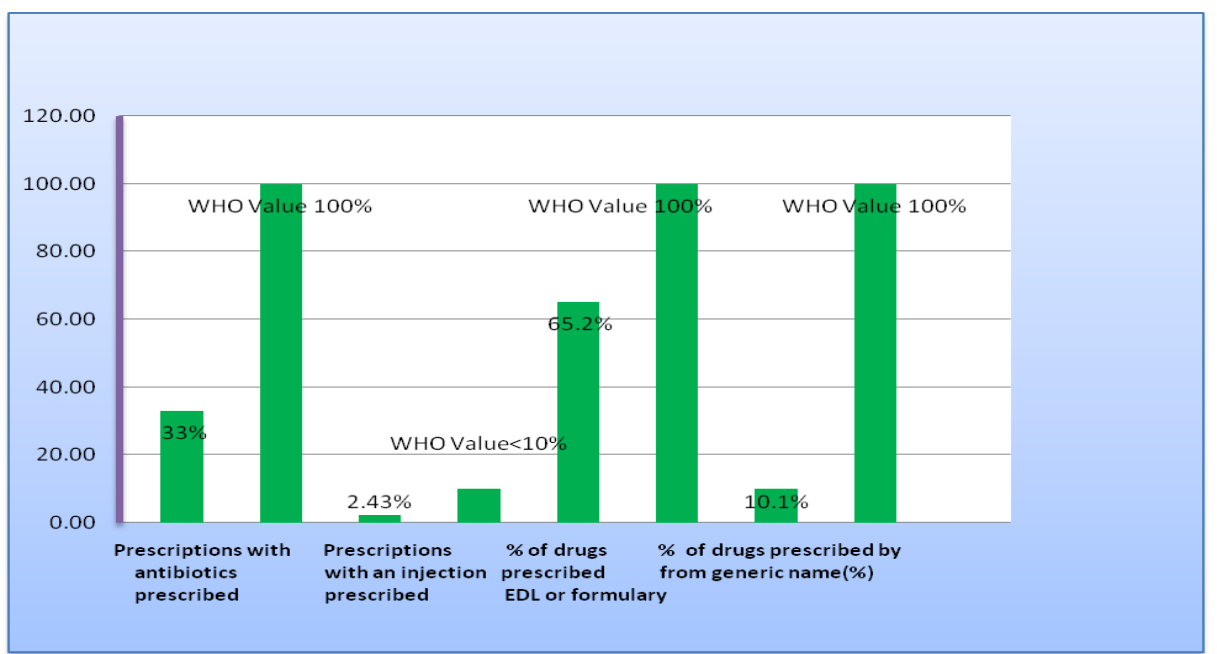

Figure 1: Bar diagram showing comparison of values of prescribing indicators of this study with WHO/INRUD prescribed values.

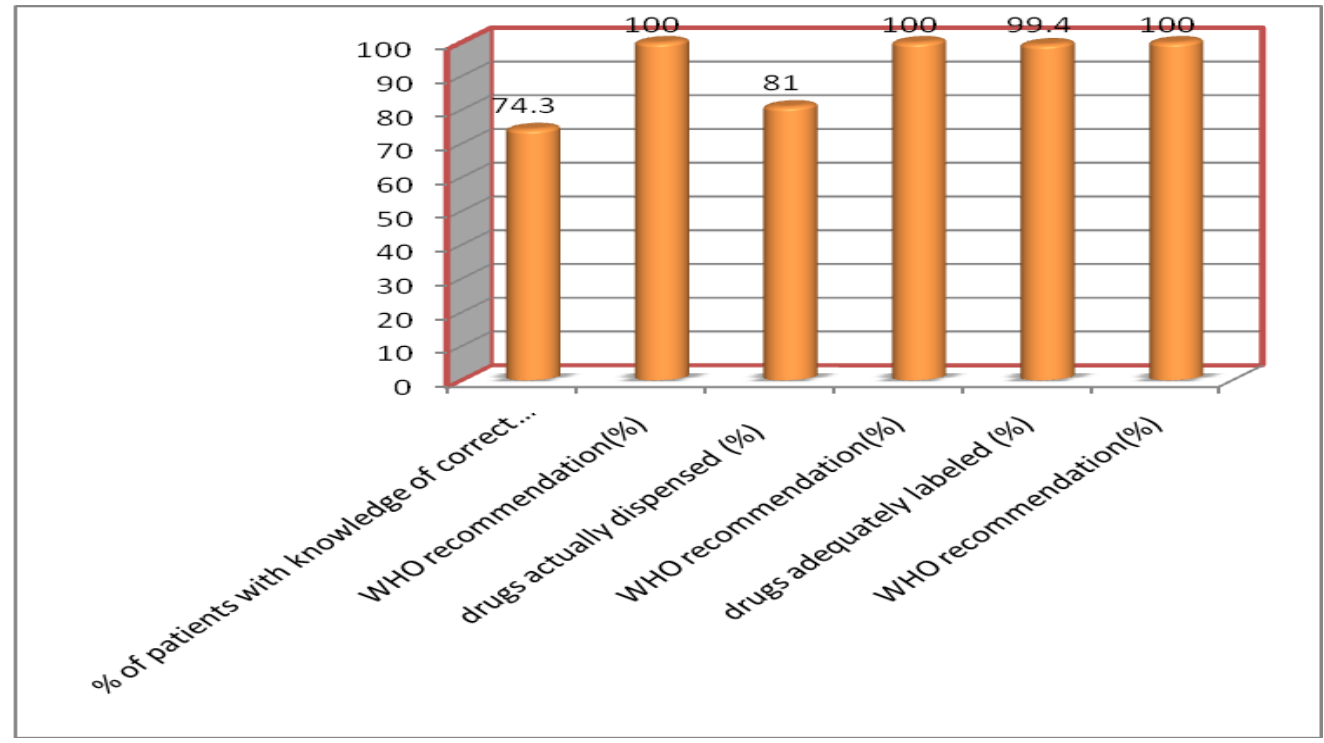

Figure 2 : Bar diagram showing comparison of values of some patient care indicators of this study with WHO/INRUD prescribed values. 
Table-1 : The distribution of the prescribing indicators in the CGHS Dispensaries at Kolkata. $(n=412)$

\begin{tabular}{|lc|}
\hline Prescribing indicators & Results \\
\hline $\begin{array}{l}\text { 1)Average no of drugs } \\
\text { per prescription } \\
+/-2 \text { S.D(range) }\end{array}$ & $3.05+/-2.54(1-7)$ \\
$\begin{array}{l}\text { 2) Prescriptions with } \\
\text { antibiotics prescribed (\%) }\end{array}$ & $136(33.0 \%)$ \\
$\begin{array}{l}\text { 3) Prescriptions with an injection } \\
\text { prescribed (\%) }\end{array}$ & $10(2.43 \%)$ \\
$\begin{array}{l}\text { 4) No. of drugs prescribed from } \\
\text { EDL or formulary (\%)* }\end{array}$ & $911(72.4 \%)$ \\
$\begin{array}{l}\text { 5) No. of drugs prescribed } \\
\text { by generic name(\%)* }\end{array}$ & $131(10.4 \%)$ \\
\hline
\end{tabular}

* ( $\%$ based on total number of drugs prescribed which was 1258)

Table-2: The distribution of the patient care indicators in the CGHS Dispensaries at Kolkata

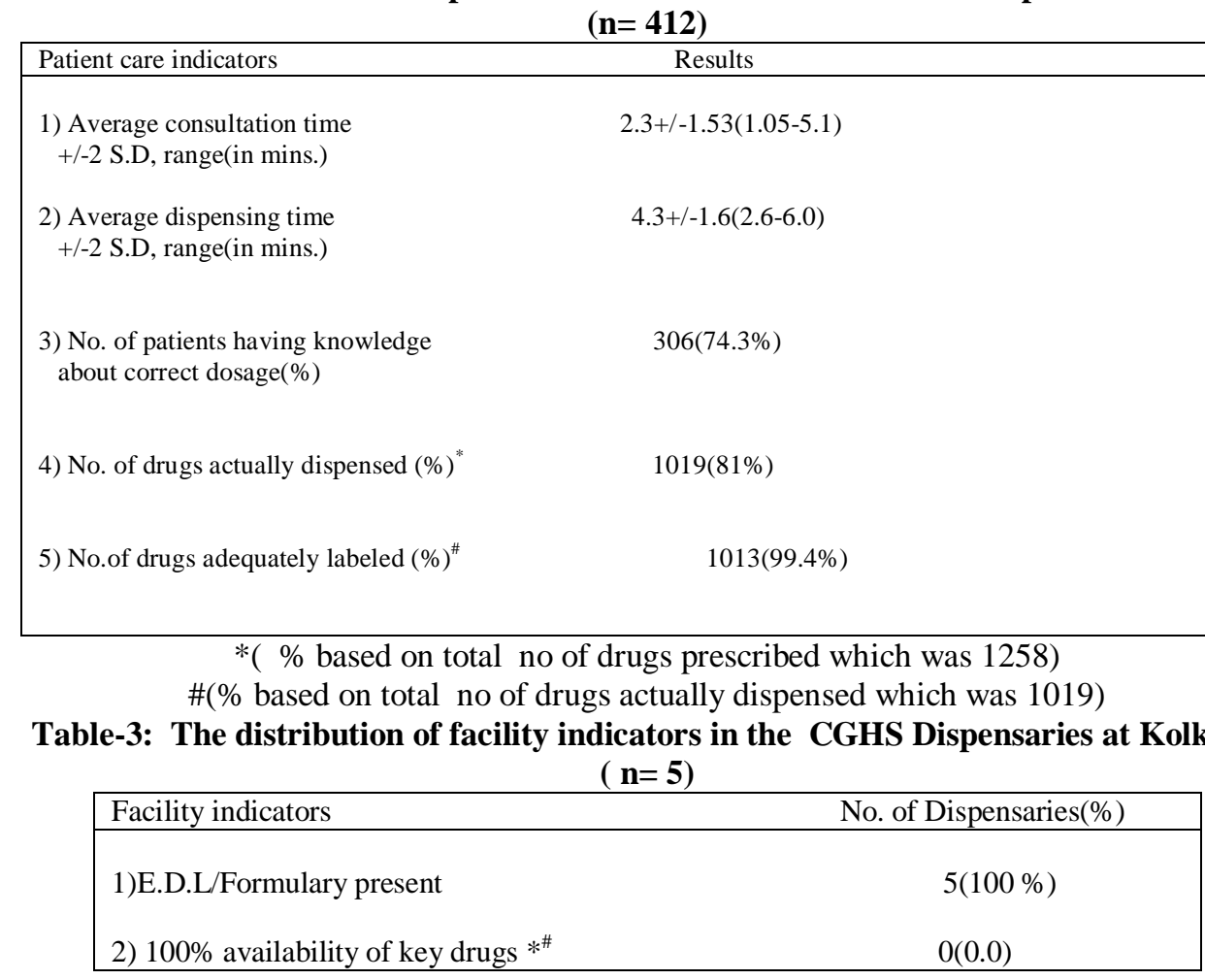

*( key drugs included Cotrimoxazole tab/syr, O.R.S, cap tetracycline, Chloroquin tab/syr, I.F.A tab/any other hematinic, Mebendazole tab/syr, PCM/other analgesics tab/syr, Antifungal tab/oint, Iodine/gentian violet/local alternative, inj t.toxoid, Tetracycline eye ointment/ other eye oint./drop)

\# (3 dispensaries had 80\% availability of key drugs and 2 dispensaries had $90 \%$ availability) 
Table-4: The distribution of some additional indicators in the 5 selected CGHS Dispensaries at Kolkata. $(\mathrm{n}=\mathbf{4 1 2})$

\begin{tabular}{|c|c|}
\hline Additional indicators & Results \\
\hline $\begin{array}{l}\text { 1) The average number of antibiotics } \\
\text { per prescription }+/-2 \text { S.D, (range) }\end{array}$ & $0.72+/-2.27(0-3)$ \\
\hline $\begin{array}{l}\text { 2) No. of prescription with } \\
\text { proper history taken }(\%)\end{array}$ & $36(8.7 \%)$ \\
\hline 3) No. of prescription with diagnosis written (\%) & $34(8.3 \%)$ \\
\hline 4) No. of prescription with referrals to higher centers (\%) & $59(14.3 \%)$ \\
\hline 5) No. of prescription with dietary advices (\%) & $42(10.2 \%)$ \\
\hline 6) No. of prescription with antiulcer $\operatorname{drugs}(\%)$ & $124(30.1 \%)$ \\
\hline 7) No. of prescription with vitamins(\%) & $105(25.5 \%)$ \\
\hline 8) No. of prescription with oral Hypoglycemics(\%) & $79(19.2 \%)$ \\
\hline 9) No. of prescription with Anti hypertensives(\%) & $113(27.4 \%)$ \\
\hline 10) No. of prescription with NSAIDS(\%) & $65(15.8 \%)$ \\
\hline 11) No. of prescription with Sedatives(\%) & $55(13.3 \%)$ \\
\hline $\begin{array}{l}\text { 12) Average cost per prescription } \\
\text { (in Rs.) }+/-2 \text { S.D,(range) }\end{array}$ & $\begin{array}{l}103.8+/-233.4 \\
(0-865)\end{array}$ \\
\hline $\begin{array}{l}\text { 13) No. of antibiotics among total number } \\
\text { of drugs prescribed } *\end{array}$ & $298(23.7 \%)$ \\
\hline $\begin{array}{l}\text { 14) The commonest antibiotics prescribed } \\
(\%)\end{array}$ & $\begin{array}{l}\text { Cotrimoxazole } \\
48(16.1 \%)\end{array}$ \\
\hline
\end{tabular}

*( \% based on total no of drugs prescribed which was 1258)

\#( $\%$ based on total no of antibiotics prescribe which was 298) 